\title{
Symmetry breaking of decaying magnetohydrodynamic Taylor-Green flows and consequences for universality
}

\author{
V. Dallas* and A. Alexakis \\ Laboratoire de Physique Statistique, École Normale Supérieure, Université Pierre et Marié Curie, Université Paris Diderot, \\ CNRS, 24 rue Lhomond, 75005 Paris, France \\ (Received 2 October 2013; published 26 December 2013)
}

\begin{abstract}
We investigate the evolution and stability of a decaying magnetohydrodynamic Taylor-Green flow, using pseudospectral simulations with resolutions up to $2048^{3}$. The chosen flow has been shown to result in a steep total energy spectrum with power law behavior $k^{-2}$. We study the symmetry breaking of this flow by exciting perturbations of different amplitudes. It is shown that for any finite amplitude perturbation there is a high enough Reynolds number for which the perturbation will grow enough at the peak of dissipation rate resulting in a nonlinear feedback into the flow and subsequently break the Taylor-Green symmetries. In particular, we show that symmetry breaking at large scales occurs if the amplitude of the perturbation is $\sigma_{\text {crit }} \sim \operatorname{Re}^{-1}$ and at small scales occurs if $\sigma_{\text {crit }} \sim \operatorname{Re}^{-3 / 2}$. This symmetry breaking modifies the scaling laws of the energy spectra at the peak of dissipation rate away from the $k^{-2}$ scaling and towards the classical $k^{-5 / 3}$ and $k^{-3 / 2}$ power laws.
\end{abstract}

DOI: 10.1103/PhysRevE.88.063017

PACS number(s): 47.27.Jv, 47.35.Tv, 47.65.-d

\section{INTRODUCTION}

In magnetohydrodynamic (MHD) turbulence several phenomenological theories exist debating the interpretation of the power law of the energy spectrum [1-5]. In summary, the power law scaling exponents obtained in these phenomenologies based on weak and strong turbulence arguments both for isotropic and anisotropic energy spectra are $-2,-5 / 3$, and $-3 / 2$. Numerical simulations to date are unable to provide a definitive answer to this scaling. For example, some direct numerical simulations (DNS) obtained energy spectra with $k^{-5 / 3}$ while others $k^{-3 / 2}$ scaling for freely decaying MHD turbulent flows [6,7]. Astrophysical observations have shown that this difference in the power law scaling also exists for the measured energy spectra of the solar wind [8]. In addition, indications of $k^{-2}$ scaling are reported for the magnetic energy spectrum measured in the magnetosphere of Jupiter [9].

Universality in MHD has been questioned by many authors [10-13] due to weak locality, anisotropy, and the possibility of various alignments that could alter the cascade mechanism. Recently, high-resolution simulations by Lee et al. [14] demonstrated $k^{-2}, k^{-5 / 3}$, and $k^{-3 / 2}$ total energy spectrum scalings for different initial conditions of the magnetic field. Thus, they showed dependence of the energy spectrum at the peak of dissipation on the initial conditions. Consequently, this suggests that all three exponents appear for different classes of flows, and hence lack of universality in decaying MHD. However, very large scale separations are needed to distinguish these power laws from any finite Reynolds number behavior [15]. The difference between $-5 / 3$ and $-3 / 2$ power laws is subtle enough (10\% difference) so that an inertial range of more than an order of magnitude is necessary to make a clear distinction between them. However, a -2 scaling exponent can be more transparent even for moderate Reynolds numbers, such as those obtained by DNS. For this reason, in this work we focus on the initial conditions that lead to

*vassilios.dallas@1ps.ens.fr the $k^{-2}$ spectrum. This scaling of the total energy spectrum was demonstrated to originate from high-shearing regions that manifest discontinuities in the magnetic field corresponding to strong current sheets [16].

All the initial conditions in [14] were satisfying symmetries of the Taylor-Green (TG) vortex [17]. This property was taken into account by numerically enforcing these symmetries in order to achieve higher resolutions with less computational cost $[14,18]$. The -2 power law spectrum was also confirmed by Dallas and Alexakis [16,19] without imposing the TG symmetries, allowing thus the turbulence to evolve freely with the view that the initial TG vortex symmetries will break at high enough Reynolds numbers. Even for their highest Taylor Reynolds number simulations $\left[\sim O\left(10^{2}\right)\right]$, the TG vortex symmetries did not break within the time interval of reaching the peak of dissipation. This suggests that the TG symmetries are a strong property of the evolution equations preserved in time. However, Stawarz et al. [20] showed that the TG symmetries can be broken at very long time scales using runs of low Reynolds numbers due to round-off error noise.

Preservation of the TG symmetries hinders the flow from exploring all phase space and consequently prevents it from reaching a universal behavior. Moreover, the breaking of the TG symmetries can possibly modify the scaling of the energy spectrum by the time of maximum dissipation rate $t_{\text {peak }}$, where the largest inertial range is obtained. Thus, before claiming lack of universality of spectral exponents for decaying MHD turbulence in periodic boxes, the persistence of the TG symmetries within $t_{\text {peak }}$ is an important issue that needs to be resolved.

We expect a critical perturbation amplitude to exist so that the system transitions from symmetry preservation to symmetry breaking within $t_{\text {peak }}$. The dependence of this critical amplitude on the Reynolds number and whether the breaking of the TG symmetries leads to a different spectral exponent are the key open questions that we address in this work. In order to demonstrate lack of universality at the peak of dissipation one needs to show that, at $\operatorname{Re} \gg 1$, there is a finite perturbation 
amplitude below which the power law of the spectrum remains unchanged, showing that the set of initial conditions which leads to a specific behavior is of nonzero measure in the limit of $\operatorname{Re} \rightarrow \infty$.

In summary, given an infinitesimal perturbation, is there a Reynolds number such that the symmetries break within $t_{\text {peak }}$ ? Will the breaking of the symmetries lead to a different power law spectrum? Are the discontinuities, which are responsible for the $k^{-2}$ spectra, formed due to enforcement of the TG symmetries? Are there universality classes for moderate Reynolds numbers or is there a universal power law scaling for the high Reynolds number limit? In this work, we investigate these questions by considering a large set of numerical simulations.

The paper is structured as follows. Section II describes the numerical methodology to solve the governing equations for our decaying MHD turbulent flows, and Sec. III provides the necessary details with regard to the Taylor-Green vortex, its symmetries, and the measures of symmetry breaking. In Sec. IV, we define our numerical parameters along with our perturbed initial conditions. First, we analyze the results from the growth of infinitesimal perturbations (see Sec. V) and then from the finite amplitude perturbations (see Sec. VI) by applying the measures of symmetry breaking. Finally, in Sec. VII we conclude by summarizing our findings.

\section{DNS OF DECAYING MHD TURBULENCE}

We consider the three-dimensional, incompressible MHD equations of fluid velocity $\boldsymbol{u}$ and magnetic induction $\boldsymbol{b}$ to be

$$
\begin{gathered}
\partial_{t} \boldsymbol{u}-(\boldsymbol{u} \times \boldsymbol{\omega})=-\nabla P+v \boldsymbol{\Delta} \boldsymbol{u}+(\boldsymbol{j} \times \boldsymbol{b}), \\
\partial_{t} \boldsymbol{b}+(\boldsymbol{u} \cdot \nabla) \boldsymbol{b}=(\boldsymbol{b} \cdot \nabla) \boldsymbol{u}+\kappa \boldsymbol{\Delta} \boldsymbol{b}, \\
\nabla \cdot \boldsymbol{u}=\nabla \cdot \boldsymbol{b}=0,
\end{gathered}
$$

with $v$ the kinematic viscosity, $\kappa$ the magnetic diffusivity, $\boldsymbol{\omega} \equiv \boldsymbol{\nabla} \times \boldsymbol{u}$ the vorticity, $\boldsymbol{j} \equiv \boldsymbol{\nabla} \times \boldsymbol{b}$ the current density, and $P=p / \rho+\frac{1}{2} \boldsymbol{u}^{2}$ the fluid pressure, composed by the plasma pressure $p$ divided by $\rho$ the constant mass density plus the hydrodynamic pressure $\frac{1}{2} \boldsymbol{u}^{2}$. If $v=\kappa=0$, the total energy $E_{t} \equiv \frac{1}{2}\left\langle|\boldsymbol{u}|^{2}+|\boldsymbol{b}|^{2}\right\rangle=E_{u}+E_{b}$, the magnetic helicity $H_{b} \equiv$ $\langle\boldsymbol{u} \cdot \boldsymbol{b}\rangle$, and the cross helicity $H_{c} \equiv\langle\boldsymbol{a} \cdot \boldsymbol{b}\rangle$ are conserved in time (the angle brackets $\langle$.$\rangle denote spatial averages in this$ study). Here, $\boldsymbol{a}$ is the magnetic potential, which is defined as $\boldsymbol{a} \equiv-\nabla^{-2}(\nabla \times b)$, since one can set $\boldsymbol{b} \equiv \boldsymbol{\nabla} \times \boldsymbol{a}$ with $\nabla \cdot \boldsymbol{a}=0$.

Our numerical method is pseudospectral [21], where each component of $\boldsymbol{u}$ and $\boldsymbol{b}$ is represented as truncated Galerkin expansions in terms of the Fourier basis using double precision accuracy. The nonlinear terms are initially computed in physical space and then transformed to spectral space using fast Fourier transforms [22]. Aliasing errors are removed using the $2 / 3$ dealiasing rule, i.e., wave numbers $k \in[1, N / 3]$, where $N$ is the number of grid points in each Cartesian coordinate of our box of period $2 \pi$. The nonlinear terms along with the pressure term are computed in such a way that $\boldsymbol{u}$ and $\boldsymbol{b}$ are projected onto a divergence-free space so that Eqs. (3) are satisfied [23].
The temporal integration of Eqs. (1) and (2) is performed using a second-order Runge-Kutta method. The code is parallelized using a hybrid parallelization (MPI-OpenMP) scheme [24].

\section{TAYLOR-GREEN VORTEX, SYMMETRIES, AND MEASURES OF SYMMETRY BREAKING}

The Taylor-Green vortex [25] is a model flow that has been used in various studies in hydrodynamics [26], dynamo [27], and anisotropic MHD [28]. The initial condition that we choose to focus on in this study is a magnetic TG flow, which results in $k^{-2}$ spectra at the peak of dissipation $[14,16,19,29]$. In particular, the initial velocity field is the Taylor-Green vortex [25] defined as

$$
\boldsymbol{u}_{T G}(\boldsymbol{x})=u_{0}\left(\begin{array}{c}
\sin x \cos y \cos z \\
-\cos x \sin y \cos z \\
0
\end{array}\right),
$$

and the initial magnetic field is given by

$$
\boldsymbol{b}_{T G}(\boldsymbol{x})=b_{0}\left(\begin{array}{c}
\cos x \sin y \sin z \\
\sin x \cos y \sin z \\
-2 \sin x \sin y \cos z
\end{array}\right),
$$

where $b_{0}$ and $u_{0}$ were chosen so that the norm of the two fields is unity; i.e., $\left\|\boldsymbol{u}_{T G}\right\|=\left\|\boldsymbol{b}_{T G}\right\|=1$. Here $\|$. $\|$ stands for the $L_{2}$ norm $\|\boldsymbol{g}\|^{2}=\frac{1}{V} \int_{V} \boldsymbol{g} \cdot \boldsymbol{g} d^{3} x$, where $\boldsymbol{g}$ is an arbitrary vector field.

Given these initial conditions and in the absence of any noise the symmetries are preserved by the evolution equations exactly $[18,19]$. In particular, we have reflection (anti)symmetries about the planes $x=0, x=\pi, y=0, y=$ $\pi, z=0$, and $z=\pi$ as well as rotational (anti)symmetries of angle $n \pi$ about the axes $(x, y, z)=\left(\frac{\pi}{2}, y, \frac{\pi}{2}\right)$ and $\left(x, \frac{\pi}{2}, \frac{\pi}{2}\right)$ and of angle $n \pi / 2$ about the axis $\left(\frac{\pi}{2}, \frac{\pi}{2}, z\right)$ for $n \in \mathbb{Z}$. The above mentioned planes that possess reflection symmetries form the insulating faces of the subboxes $[0, \pi]^{3}[17]$, where the $\boldsymbol{j}_{T G}$ is everywhere parallel to these faces. Note that for these particular initial conditions $\boldsymbol{b}_{T G}$ satisfies the same symmetries with $\boldsymbol{\omega}_{T G}$ and $\boldsymbol{u}_{T G}$ with $\boldsymbol{j}_{T G}$.

It was shown in [16] that the $k^{-2}$ spectrum observed in the numerical simulations originates from the formation of strong current sheets at the reflection symmetry planes $x=0, x=\pi, y=0$, and $y=\pi$. So, we focus on only one of these symmetries. In particular, we will investigate the reflection symmetry around the plane $x=0$. We then define the reflection operator $\boldsymbol{R}_{x}$ around the $x=0$ plane as

$$
\boldsymbol{R}_{x}\left(\begin{array}{l}
g_{x}(x, y, z) \\
g_{y}(x, y, z) \\
g_{z}(x, y, z)
\end{array}\right)=\left(\begin{array}{c}
-g_{x}(-x, y, z) \\
g_{y}(-x, y, z) \\
g_{z}(-x, y, z)
\end{array}\right) .
$$

The TG initial conditions under the action of $\boldsymbol{R}_{x}$ transform as follows:

$$
\boldsymbol{R}_{x} \boldsymbol{u}_{T G}=\boldsymbol{u}_{T G}, \quad \boldsymbol{R}_{x} \boldsymbol{b}_{T G}=-\boldsymbol{b}_{T G} .
$$

Given any arbitrary set of fields $\boldsymbol{u}, \boldsymbol{b}$ we define $\boldsymbol{u}_{s}$ and $\boldsymbol{b}_{s}$ as

$$
\boldsymbol{u}_{s}=\frac{1}{2}\left(\boldsymbol{u}+\boldsymbol{R}_{x} \boldsymbol{u}\right), \quad \boldsymbol{b}_{s}=\frac{1}{2}\left(\boldsymbol{b}-\boldsymbol{R}_{x} \boldsymbol{b}\right)
$$

with $\boldsymbol{u}_{s}$ and $\boldsymbol{b}_{s}$ transforming similarly to the TG initial conditions under reflection $\boldsymbol{R}_{x}$ [see Eq. (7)]. Similarly we 
define $\boldsymbol{u}_{a}$ and $\boldsymbol{b}_{a}$

$$
\boldsymbol{u}_{a}=\frac{1}{2}\left(\boldsymbol{u}-\boldsymbol{R}_{x} \boldsymbol{u}\right), \quad \boldsymbol{b}_{a}=\frac{1}{2}\left(\boldsymbol{b}+\boldsymbol{R}_{x} \boldsymbol{b}\right)
$$

as the part of the flow that does not follow the TG symmetries. Note that $\boldsymbol{u}_{a}$ and $\boldsymbol{b}_{a}$ transform differently under reflection; i.e.,

$$
\boldsymbol{R}_{x} \boldsymbol{u}_{a}=-\boldsymbol{u}_{a}, \quad \boldsymbol{R}_{x} \boldsymbol{b}_{a}=\boldsymbol{b}_{a} .
$$

We will refer to $\boldsymbol{u}_{s}, \boldsymbol{b}_{s}$ as the symmetric part of the flow while to $\boldsymbol{u}_{a}, \boldsymbol{b}_{a}$ as the asymmetric part of the flow. Note that if we start with $\boldsymbol{u}=\boldsymbol{u}_{T G}$ and $\boldsymbol{b}=\boldsymbol{b}_{T G}$ at $t=0$, then $\boldsymbol{u}_{a}, \boldsymbol{b}_{a}$ will remain zero throughout the computation. Thus, $\boldsymbol{u}_{a}, \boldsymbol{b}_{a}$ can provide us with a measure of the extent to which the symmetries are broken. Here we will focus on two such measures. First we consider the ratio of the energies of asymmetric to the symmetric component of the fields $E_{a} / E_{s}$, where

$$
\begin{aligned}
& E_{a}=\frac{1}{2}\left(\left\|\boldsymbol{u}_{a}\right\|^{2}+\left\|\boldsymbol{b}_{a}\right\|^{2}\right), \\
& E_{s}=\frac{1}{2}\left(\left\|\boldsymbol{u}_{s}\right\|^{2}+\left\|\boldsymbol{b}_{s}\right\|^{2}\right) .
\end{aligned}
$$

This quantity provides a measure of the degree to which the TG symmetries are broken in the large (energy containing) scales. From Eqs. (9) we have the total energy $E_{t}=E_{s}+E_{a}$. We also focus on the small scales by looking at the ratio of the dissipation rates $\epsilon_{a} / \epsilon_{s}$, where

$$
\begin{gathered}
\epsilon_{a}=v\left\|\nabla \times \boldsymbol{u}_{a}\right\|^{2}+\kappa\left\|\nabla \times \boldsymbol{b}_{a}\right\|^{2}, \\
\epsilon_{s}=v\left\|\nabla \times \boldsymbol{u}_{s}\right\|^{2}+\kappa\left\|\nabla \times \boldsymbol{b}_{s}\right\|^{2} .
\end{gathered}
$$

Similarly to the energy we have the total dissipation rate $\epsilon_{t}=$ $\epsilon_{a}+\epsilon_{s}$, where the fact that $R_{x}[\nabla \times \mathbf{u}]=-\nabla \times R_{x}[\mathbf{u}][30]$ is used to derive this last result.

\section{INITIAL CONDITIONS AND SIMULATION PARAMETERS}

To study the stability of the TG symmetries and their implications on the energy spectrum a series of numerical simulations were performed. The simulations were carried out on a triple periodic box of size $2 \pi$. The initial conditions were composed by the TG initial conditions plus small perturbation fields $\sqrt{\sigma} \boldsymbol{u}_{p}, \sqrt{\sigma} \boldsymbol{b}_{p}$, viz.

$$
\boldsymbol{u}=\boldsymbol{u}_{T G}+\sqrt{\sigma} \boldsymbol{u}_{p}, \quad \boldsymbol{b}=\boldsymbol{b}_{T G}+\sqrt{\sigma} \boldsymbol{b}_{p} .
$$

The perturbation fields $\boldsymbol{u}_{p}, \boldsymbol{b}_{p}$ were chosen to be a superposition of Fourier modes in spherical shells $2 \leqslant|\boldsymbol{k}| \leqslant k_{p}$. The phases of the Fourier modes were chosen so that $\boldsymbol{R}_{x} \boldsymbol{u}_{p}=-\boldsymbol{u}_{p}$, $\boldsymbol{R}_{x} \boldsymbol{b}_{p}=\boldsymbol{b}_{p}$ and random otherwise. In this way we guarantee that the two perturbation fields give no contribution to $\boldsymbol{u}_{s}$ and $\boldsymbol{b}_{s}$. The norm of the two fields was set to unity $\left\|\boldsymbol{u}_{p}\right\|=\left\|\boldsymbol{b}_{p}\right\|=$ 1 so that the amplitude $\sigma$ at $t=0$ expresses the ratio of the kinetic energy of the perturbation field to the energy of the TG flow (i.e., $\left.\sigma \equiv E_{a}\right|_{t=0} /\left.E_{s}\right|_{t=0}$ ). Additionally, we define $\sigma_{\epsilon} \equiv$ $\left.\epsilon_{a}\right|_{t=0} /\left.\epsilon_{s}\right|_{t=0}$ as the ratio of the dissipation rate of the asymmetric part of the flow to the symmetric part of the flow at $t=0$.

The Reynolds number is defined based on the velocity rms value at $t=0$ and smallest wave number $k_{T G}=1$ in
TABLE I. Numerical parameters of the DNS. For all runs $v=\kappa$. Note that $k_{\max }=N / 3$, using the $2 / 3$ dealiasing rule and the values of $k_{\max } \eta$ are reported at the peak of $\epsilon_{t}$.

\begin{tabular}{lcccccccc}
\hline \hline \multicolumn{2}{c}{$\begin{array}{c}\sigma=10^{-6}, k_{p}=10 \\
\sigma_{\epsilon}=1.8 \times 10^{-5}\end{array}$} & \multicolumn{3}{c}{$\sigma=0.01, k_{p}=4$} & \multicolumn{2}{c}{$\sigma=0.1, k_{p}=4$} \\
$\sigma_{\epsilon}=0.054$
\end{tabular}

the box; i.e., $\operatorname{Re} \equiv\left\|u_{T G}\right\| / \nu k_{T G}$. With these scales we can also define the eddy turnover time $\tau_{L} \equiv\left(u_{T G} k_{T G}\right)^{-1}=1$ at $t=0$. The smallest length scale in our flows is defined based on Kolmogorov scaling $\eta \equiv\left(v^{3} / \epsilon_{t}\right)^{1 / 4}$, where $\epsilon_{t}=$ $v\left\langle|\omega|^{2}\right\rangle+\kappa\left\langle|j|^{2}\right\rangle$ is the total dissipation rate of energy. In all runs $v=\kappa$ and thus the Prandtl number is always unity. The set of parameters for all the examined runs is given in Table I.

\section{GROWTH OF INFINITESIMAL PERTURBATIONS}

As a first step we look at the temporal evolution of flows with energy ratio $\sigma=10^{-6}$ and dissipation ratio $\sigma_{\epsilon}=$ $1.8 \times 10^{-5}$ at $t=0$. For this choice and for all Reynolds numbers considered here the amplitude of the perturbation (symmetry breaking part of the flow) remains much smaller than the symmetric part of the flow at all times of interest. Thus, there is negligible effect of the perturbation on the part of the flow that obeys the TG symmetries and $\boldsymbol{u}_{a}, \boldsymbol{b}_{a}$ evolve passively following the MHD equations (1) and (2) linearized around $\boldsymbol{u}_{s}, \boldsymbol{b}_{s}$.

Figure 1 shows the temporal evolution of $E_{s}$ in blue (dark gray) and $E_{a}$ in red (light gray) for the seven different Reynolds numbers examined. The lower curves are the small-Re cases while the top curves are the high-Re cases. The vertical dashed line indicates $t_{\text {peak }}$, the time that $\epsilon_{t}$ is peaked, which is the time we are interested in. In this case, very weak variations of $t_{\text {peak }}$ were observed with Re. It is evident that as the Reynolds number is increased the growth of the asymmetric part of the energy $E_{a}$ is increased.

Since we are interested in the symmetry breaking at the peak of dissipation, we plot the ratio $E_{a} / E_{s}$ at $t_{\text {peak }}$ as a function of the Reynolds number (see Fig. 2). This energy ratio appears to increase linearly with the Reynolds number. This linear increase of the perturbation energy can be understood if we consider that the main source of growth of the perturbation comes from the magnetic shear layer at the $x=0$ plane whose strength increases with Re. Following a simple twodimensional reconnection model [31], the time scale for the growth of a perturbation in such layers is controlled by the shear rate $B_{0} / \delta$ where $B_{0}$ is the amplitude of the magnetic field in the layer and $\delta$ is the thickness of the shear layer. $B_{0}$ has negligible dependence on $\mathrm{Re}$ and is determined by the initial conditions. The thickness of the reconnection layer $\delta$ 


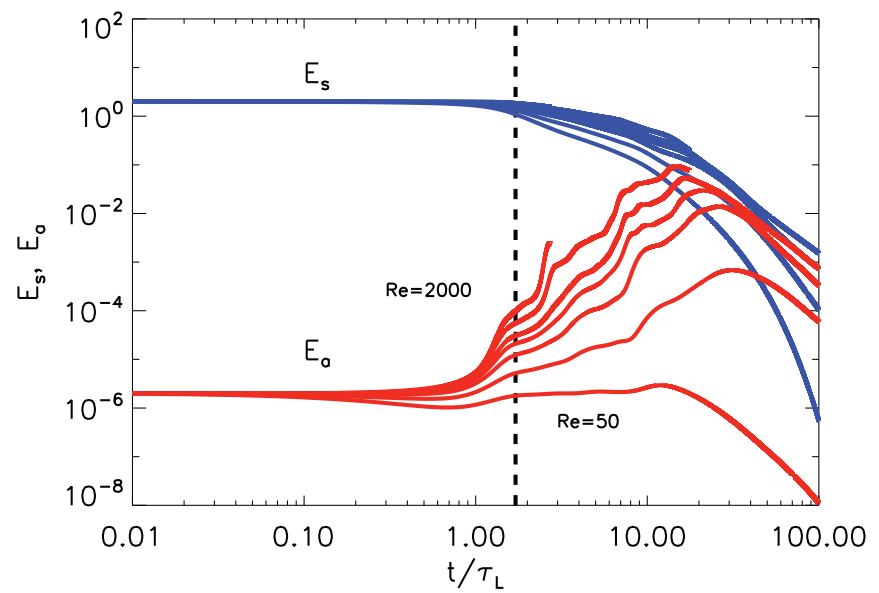

FIG. 1. (Color online) Evolution of $E_{s}$ and $E_{a}$ as a function of time for different Reynolds numbers. The vertical dashed line indicates the time of maximum total dissipation rate.

is expected to scale like $\delta \sim L / \sqrt{S_{L}}$ where $L$ is the length of the layer that is of the order of the box size and $S_{L}$ the Lundquist number $S_{L}=B_{0} L / \kappa$ [31]. For this problem $S_{L} \sim$ Re since $\|\boldsymbol{u}\| \sim\|\boldsymbol{b}\|$ and $v=\kappa$. At the short time scale $t_{\text {peak }}$ we expect that transient growth rates will dominate and the growth of the perturbation in time will be linear rather than exponential. Therefore, we expect that the amplitude of the perturbation $A_{p}$ at $t_{\text {peak }}$ will increase from the initial value $A_{0}$ as

$$
\begin{aligned}
A_{p} & \sim A_{0} \frac{B_{0}}{\delta} t_{\text {peak }} \\
& \sim A_{0} B_{0} \frac{t_{\text {peak }}}{L} \operatorname{Re}^{1 / 2},
\end{aligned}
$$

from which we conclude that

$$
\frac{E_{a}}{E_{s}} \sim \frac{A_{p}^{2}}{B_{0}^{2}} \sim A_{0}^{2}\left(\frac{t_{\text {peak }}}{L}\right)^{2} \mathrm{Re},
$$

and hence the linear increase observed in Fig. 2. This result comes from simple dimensional arguments that identify the relevant time and length scales involved. Therefore, we expect their validity even if the true three-dimensional setup can be more complex [32].

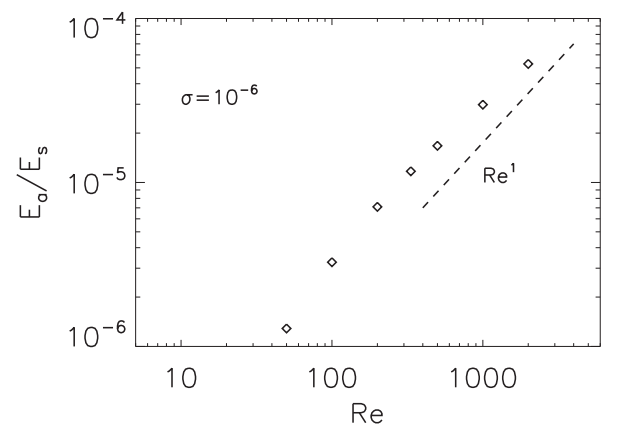

FIG. 2. Energy ratio $E_{p} / E_{s}$ at the time of maximum dissipation rate as function of the Reynolds number for $\sigma=10^{-6}$.

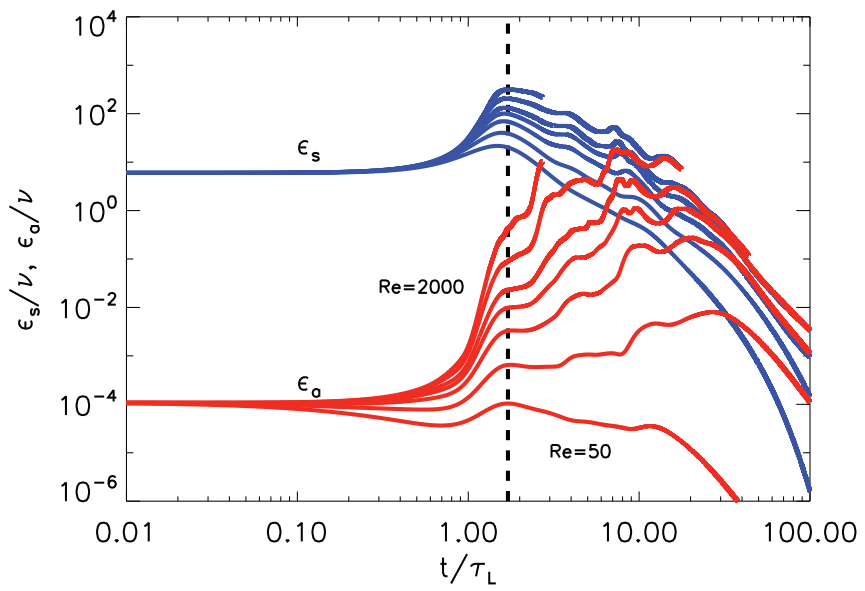

FIG. 3. (Color online) Evolution of $\epsilon_{s} / \nu$ and $\epsilon_{a} / v$ as a function of time for different Reynolds numbers. The vertical dashed line indicates the time of maximum total dissipation rate.

Figure 3 presents the time evolution of the two dissipations $\epsilon_{s}$ and $\epsilon_{a}$ divided by the viscosity $\nu$. While both grow with time, the asymmetric dissipation $\epsilon_{a} / v$ increases by roughly four orders of magnitude in two turnover times for the highest Reynolds number examined.

In Fig. 4 we plot the ratio of the two dissipations $\epsilon_{a} / \epsilon_{s}$ at $t_{\text {peak }}$. This ratio is increasing faster than linear with Reynolds number, indicating that symmetries break even faster in the small scales. The scaling observed is close to

$$
\epsilon_{a} / \epsilon_{s} \sim \mathrm{Re}^{3 / 2} .
$$

The fast breaking of the symmetries in the small scales can also be seen by looking at the energy spectra of the fields $\boldsymbol{u}_{s}, \boldsymbol{u}_{a}, \boldsymbol{b}_{s}$, and $\boldsymbol{b}_{a}$. Figures 5(a) and 5(b) show the symmetric and asymmetric part of the magnetic $\left(E_{b, s}, E_{b, a}\right)$ and the kinetic $\left(E_{u, s}, E_{u, a}\right)$ energy spectra, respectively, compensated by $k^{2}$ for different times up to $t_{\text {peak }}$. Red (dark gray) curves represent the energy spectra of the asymmetric part of the flow, while blue curves (light gray) the energy spectra of the symmetric part of the flow. The black line represents the spectrum of the total field, i.e., $E_{b}=E_{b, s}+E_{b, a}$ and $E_{u}=E_{u, s}+E_{u, a}$, at the time of the maximum dissipation rate.

It is important to note the asymmetric part of the flow is always smaller than the symmetric part for all scales. Thus,

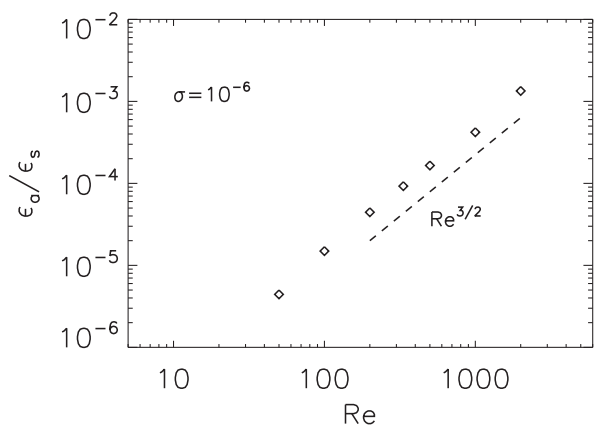

FIG. 4. Dissipation ratio $\epsilon_{p} / \epsilon_{s}$ at the time of maximum dissipation rate as function of the Reynolds number for $\sigma=10^{-6}$. 


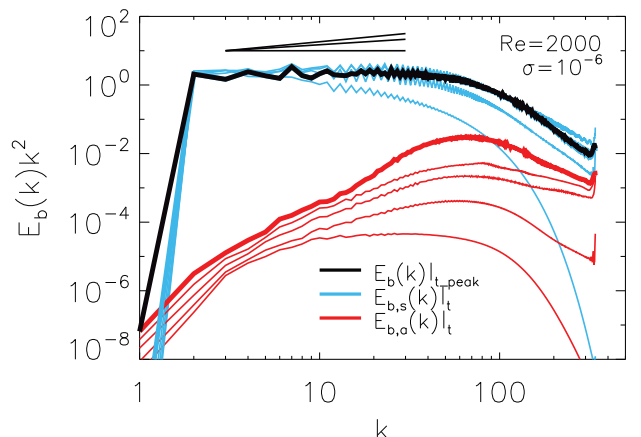

(a)

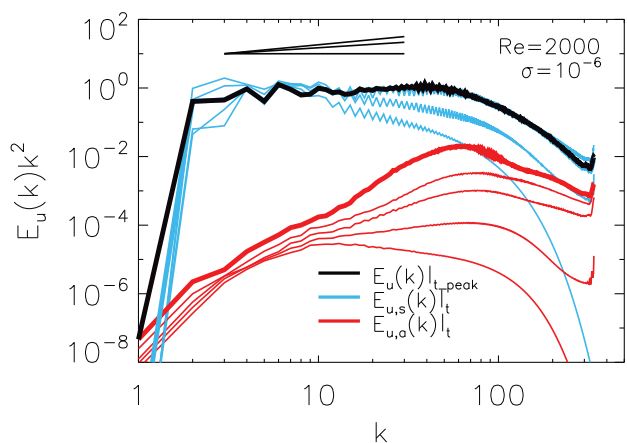

(b)

FIG. 5. (Color online) (a) Magnetic energy and (b) kinetic energy spectra compensated by $k^{2}$ for $\sigma=10^{-6}$ and $\operatorname{Re}=2000$. The blue (light gray) and red (dark gray) curves represent the symmetric and asymmetric parts of the spectrum, respectively, at different times. The black curve represents the total (i.e., sum of symmetric and asymmetric part) spectrum at $t_{\text {peak }}$. The straight lines indicate the proposed $k^{-2}, k^{-5 / 3}$, and $k^{-3 / 2}$ spectral slopes.

the symmetric part of the flow reproduces the $k^{-2}$ energy spectrum, while the asymmetric part of the spectrum evolves passively.

The results of this section show that no matter how small the amplitude $\sigma$ of the perturbation added in the TG initial conditions there is a Re for which the perturbation will grow significantly enough for it to play a (nonlinear) dynamical role in the system. This critical amplitude can be estimated from our runs to be either $\sigma_{\text {crit }}=C_{1} \mathrm{Re}^{-1}$ if energy estimates are considered or $\sigma_{\text {crit }}=C_{2} \mathrm{Re}^{-3 / 2}$ if dissipation estimates are considered, where $C_{1}$ and $C_{2}$ are constants. Consequently, the results explain why symmetry breaking was not observed at $t_{\text {peak }}$ in the simulations of $[14,16,19,29]$ due to the presence of numerical noise. Simulations using single precision accuracy introduce perturbations of amplitude $\sigma \sim 10^{-8}$ and thus $\operatorname{Re} \sim 10^{8}$ would be required for the symmetries to break in the large scales and $\operatorname{Re} \sim 10^{5}$ for the symmetries to break in the small scales. Simulations at such Reynolds numbers cannot be performed on today's largest supercomputers even at single precision accuracy. Therefore, this would make the observation of symmetry breaking by numerical noise alone impossible at $t_{\text {peak }}$. We note, however, that symmetry breaking can be observed at later times as was shown in [20].

\section{GROWTH OF FINITE AMPLITUDE PERTURBATIONS}

The growth of infinitesimal perturbations gives us a lot of information on the growth of symmetry breaking perturbations. However, it does not provide us with any information about a possible change in the spectral exponent and the return or not to a universal behavior. For this reason we have performed two series of simulations with perturbation amplitude $\sigma=0.01$ and $\sigma=0.1$ (see Table I). For these values of $\sigma$ the perturbation grows sufficiently large at $t_{\text {peak }}$ to play a dynamical role in the evolution of the flow. The wave number range of the initial conditions of $\boldsymbol{u}_{p}$ and $\boldsymbol{b}_{p}$ was limited within $2 \leqslant|\boldsymbol{k}| \leqslant 4$. The energy ratio $\sigma$ and the dissipation ratio $\sigma_{\epsilon}$ at $t=0$ are $\sigma_{\epsilon} \simeq 0.54$ for the $\sigma=0.1$ runs and $\sigma_{\epsilon} \simeq 0.054$ for the $\sigma=0.01$ runs.

\section{A. Temporal behavior}

Figure 6(a) shows the time evolution of Ohmic dissipation rate $\epsilon_{b}$ for the runs with $\mathrm{Re}=2000$ and three different values of $\sigma$. One can notice that the perturbation of amplitude $\sigma=0.1$ has significantly changed the time evolution of $\epsilon_{b}$, which has increased in amplitude and its peak has been shifted later in time (i.e., $t_{\text {peak }} / \tau_{L} \simeq 1.8$ ). Smaller variations are observed for the $\sigma=0.01$ case with a small increase of $\epsilon_{b}(\sim 5 \%)$ and no visible change in $t_{\text {peak }} / \tau_{L} \simeq 1.7$ in comparison to the

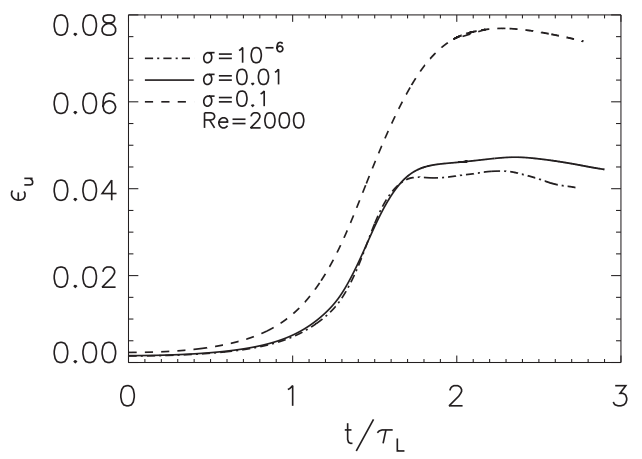

(b)

FIG. 6. (a) Ohmic and (b) viscous dissipation rate as a function of time for $\mathrm{Re}=2000$ and three different values of $\sigma$. 


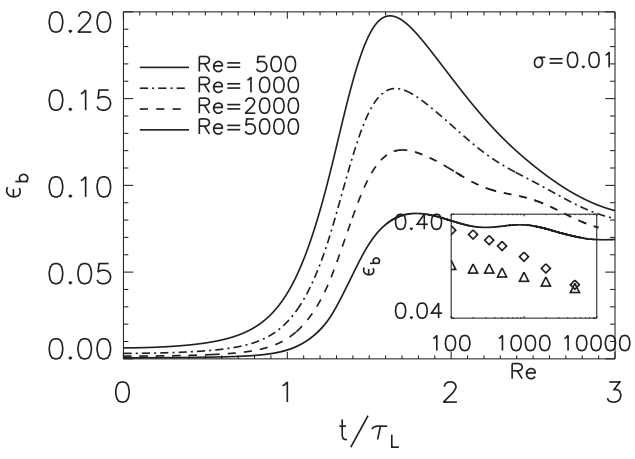

(a)

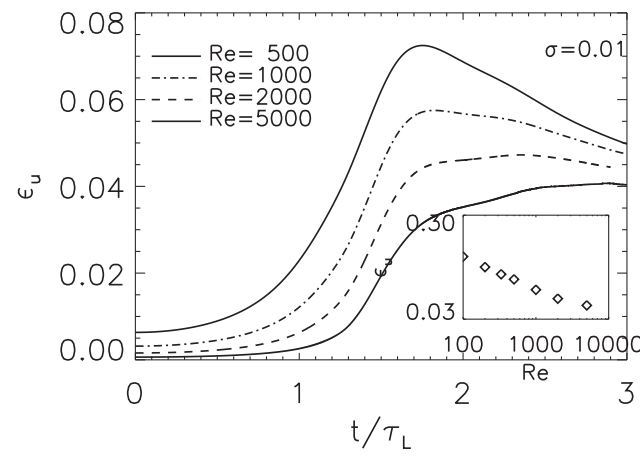

(b)

FIG. 7. (a) Ohmic and (b) viscous dissipation rate as a function of time for $\sigma=0.01$ and different values of Re.

$\sigma=10^{-6}$ case. Moreover, a new local peak starts to form around $t / \tau_{L} \simeq 2.4$ that is not present for the $\sigma=10^{-6}$ run.

In Fig. 6(b) we observe that the viscous dissipation rate $\epsilon_{u}$ peaks at later times than $\epsilon_{b}$ at this Re. For the run with perturbation amplitude $\sigma=0.1$ the peak of the viscous dissipation rate has increased by $40 \%$ while for $\sigma=0.01$ there is a $10 \%$ increase with reference to the $\sigma=10^{-6}$ case. It is worth noting that the maximum of $\epsilon_{u}$ for the $\sigma=0.01$ case coincides with the second local maximum of $\epsilon_{b}$ that takes place at $t / \tau_{L} \simeq 2.4$.

The occurrence of the new local peak of Ohmic dissipation can be seen more clearly at higher Reynolds numbers. Figure 7(a) presents the dissipation rates as a function of time for four different $\operatorname{Re}$ and $\sigma=0.01$. For small values of the Reynolds number a single peak appears for the time evolution of $\epsilon_{b}$ at $t / \tau_{L} \simeq 1.7$ that coincides with the time of the dissipation peak observed in the unperturbed system. This peak is due to the formation of large current sheets at symmetry planes [16]. As the Reynolds number is increased a new maximum appears at $t / \tau_{L} \simeq 2.4$. This new peak, as we will show later, is due to the formation of smaller scales by the breaking of the symmetries. It can be seen clearly only for the $\mathrm{Re}=5000$ run and it is very close in amplitude with the first peak at $t / \tau_{L} \simeq 1.7$. Hence, the role played by this small perturbation is only apparent at very large Re for which the current sheet becomes unstable, while its effect is muffled at smaller Re. On the other hand, a single maximum is developed in the evolution of the viscous dissipation rate $\epsilon_{u}$, which occurs at $t / \tau_{L} \simeq 1.8$ (a little later than the Ohmic dissipation maximum) for $\mathrm{Re} \leqslant 1000$ [see Fig. 7(b)] but moves farther in time at $t / \tau_{L} \simeq 2.4$ and 2.9 for $\mathrm{Re}=2000$ and 5000, respectively.

The inset in Fig. 7(a) illustrates the values of $\epsilon_{b}$ at $t / \tau_{L}=$ 1.7 (diamonds) and at $t / \tau_{L}=2.4$ (triangles) for different Reynolds numbers. The second peak of $\epsilon_{b}$ seems to reach each asymptotic state much faster than the first peak as $\mathrm{Re}$ increases with $\mathrm{Re}=5000$ the transitional point where $\left.\left.\epsilon_{b}\right|_{t / \tau_{L}=1.7} \simeq \epsilon_{b}\right|_{t / \tau_{L}=2.4}$. Then, for $\operatorname{Re} \gg 1$ the trends of the two peaks of $\epsilon_{b}$ suggest that the second peak will become dominant and Reynolds number independent. The maximum values of $\epsilon_{u}$ that are plotted in the inset of Fig. 7(b) as a function of $\operatorname{Re}$ also indicate that the viscous dissipation rate is far from reaching its asymptotic state even for our highest resolution simulations $(\mathrm{Re}=5000)$, which are at the limit of the current computational power. It is interesting that the Ohmic and viscous dissipation obey different high Reynolds number asymptotics. In other words, the small scales of the magnetic field seem to reach its universal regime at lower $\mathrm{Re}$ than the small scales of the velocity field.

\section{B. Symmetry breaking and structures}

In Figs. 8(a) and 8(b) we show the values of the energy ratio $E_{a} / E_{s}$ and the dissipation ratio $\epsilon_{a} / \epsilon_{s}$, respectively, at the peak of the energy dissipation rate for all the different $\operatorname{Re}$ that we consider in this study for runs with $\sigma=0.01$ and 0.1 (see Table I). The effect of nonlinearity is evident, since both cases

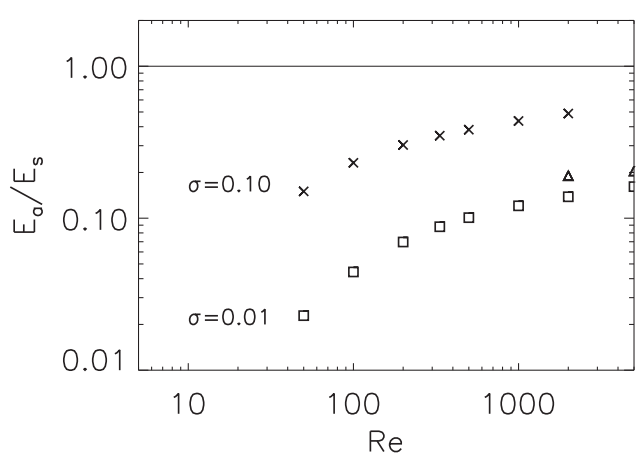

(a)

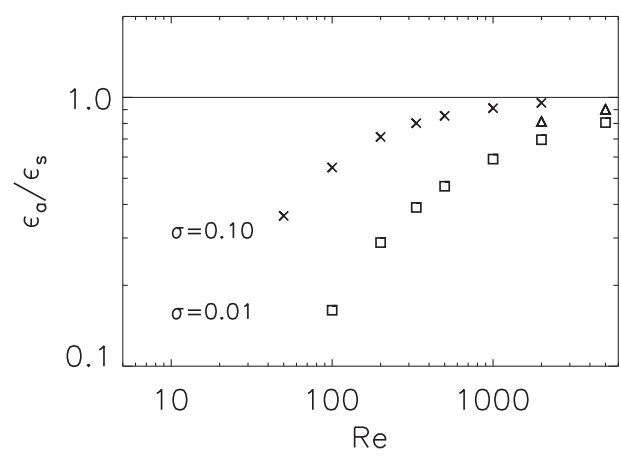

(b)

FIG. 8. (a) Energy ratio $E_{a} / E_{s}$ and (b) dissipation ratio $\epsilon_{a} / \epsilon_{s}$ as functions of Re at the time of maximum dissipation rate for $\sigma=0.01$ and 0.1 . The triangles correspond to the run with $\sigma=0.01$ for the second peak of dissipation that only appeared at the high-Re cases at $t / \tau_{L} \simeq 2.4$. 

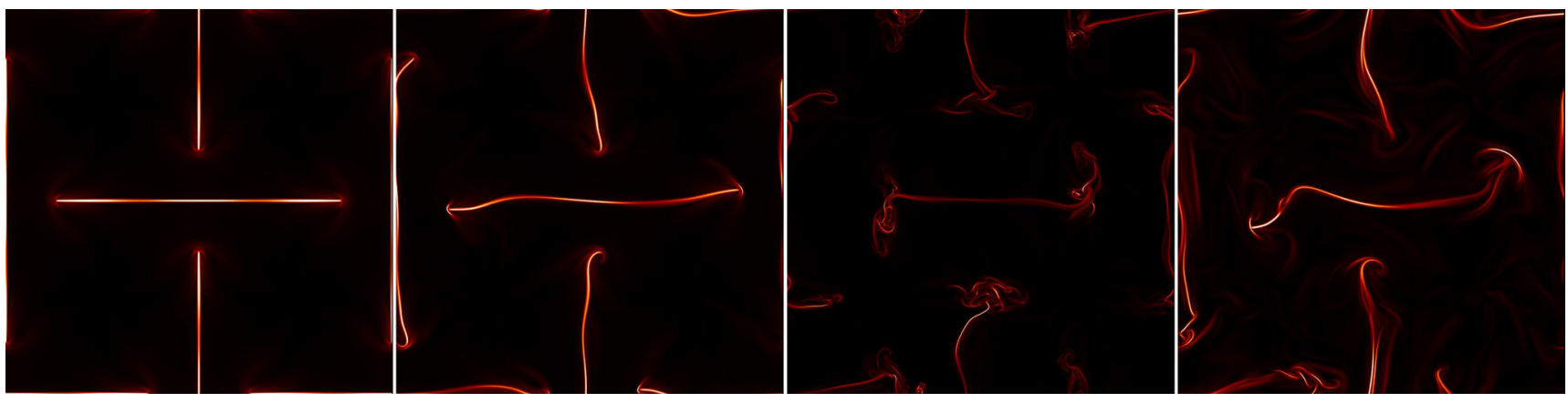

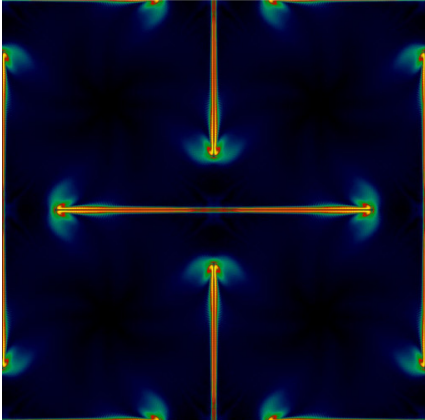

(a)

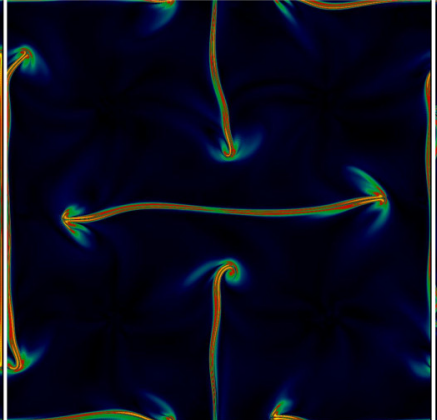

(b)

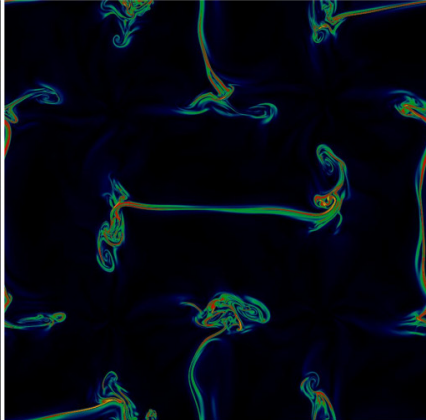

(c)

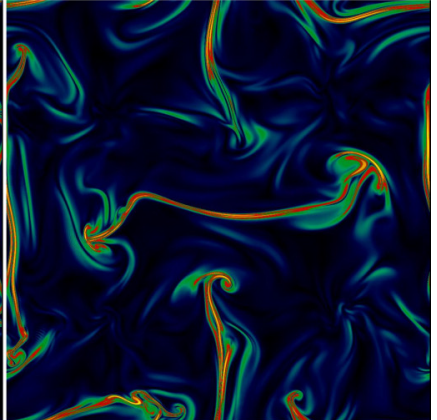

(d)

FIG. 9. (Color online) Current (top panels) and vorticity (bottom panels) density at the time of maximum dissipation rate for (a) $\sigma=10^{-6}$, $\operatorname{Re}=2000 ;$ (b) $\sigma=0.01, \operatorname{Re}=2000 ;$ (c) $\sigma=0.01, \operatorname{Re}=5000$; and (d) $\sigma=0.1, \operatorname{Re}=2000$.

deviate from the scalings $E_{a} / E_{s} \sim \operatorname{Re}$ and $\epsilon_{a} / \epsilon_{s} \sim \operatorname{Re}^{3 / 2}$ observed in Sec. V. In particular, at high Re the asymmetric part of the energy for the runs with $\sigma=0.1$ appears to asymptote towards $E_{a} \simeq 0.5 E_{s}$ while in the $\sigma=0.01$ it is significantly smaller, i.e., $E_{a} \simeq 0.15 E_{s}$, with slightly higher value at the second dissipation peak [see Fig. 8(a)]. This implies that at the large, energy containing scales only a modest breaking of the symmetries has occurred for $\sigma=0.01$ runs. In the small scales, however, the symmetries seem to be fully broken for both values of $\sigma$. For the $\sigma=0.1$ case the dissipation ratio reaches values close to unity even for $\operatorname{Re}=500$ and for $\sigma=0.01$ we have $\epsilon_{a} \simeq 0.8 \epsilon_{s}$ at the first dissipation peak and $\epsilon_{a} \simeq 0.9 \epsilon_{s}$ at the second dissipation peak for $\mathrm{Re}=5000$.

Symmetry breaking of the resulting structures can be also realized through visualizations. While three-dimensional images of the full computational box provide global information, they are sensitive in the choice of isocontour levels and very often can be misleading. We have thus chosen to show color plots of two-dimensional slices that pass through the high current and vorticity density regions.

Figure 9 illustrates the current (top panels) and vorticity (bottom panels) density at the $z=\pi / 4$ plane. In Fig. 9(a) we show results for the $\sigma=10^{-6}$ and $\operatorname{Re}=2000$ run where the perturbation evolved passively. In this case, the strong current and vorticity sheets that appear at the $x=0, \pi / 2$ and $y=0, \pi / 2$ planes are responsible for the $k^{-2}$ energy spectrum (see [16]). Therefore, the stability of these structures is crucial to determine the presence or absence of universality. The effect of the perturbation on the structures becomes pronounced for the $\sigma=10^{-2}$ and $\operatorname{Re}=2000$ case [see Fig. 9(b)]. The

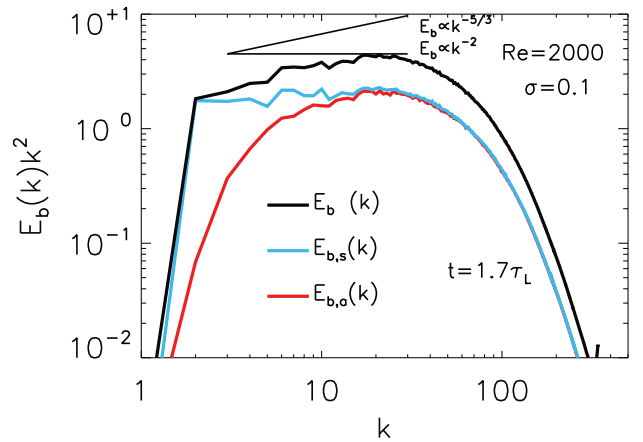

(a)

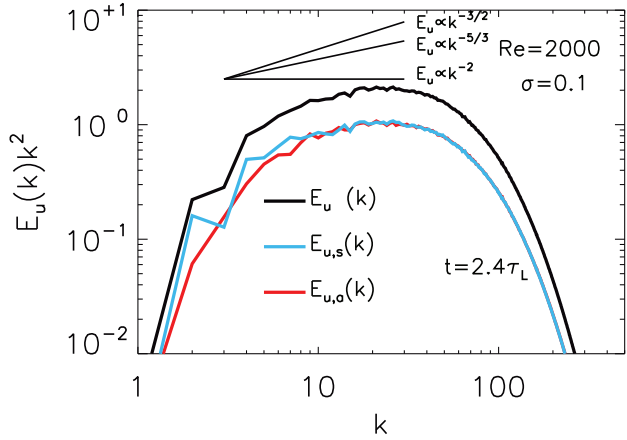

(b)

FIG. 10. (Color online) (a) Magnetic energy and (b) kinetic energy spectra at the peak of Ohmic and viscous dissipation rate, respectively, for $\sigma=0.1$ and $\operatorname{Re}=2000$. 


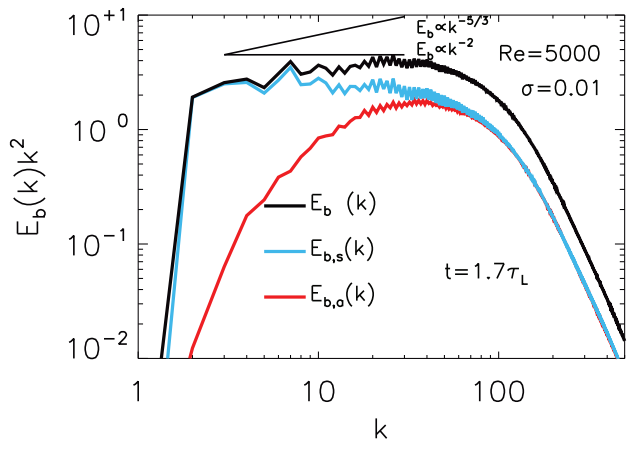

(a)

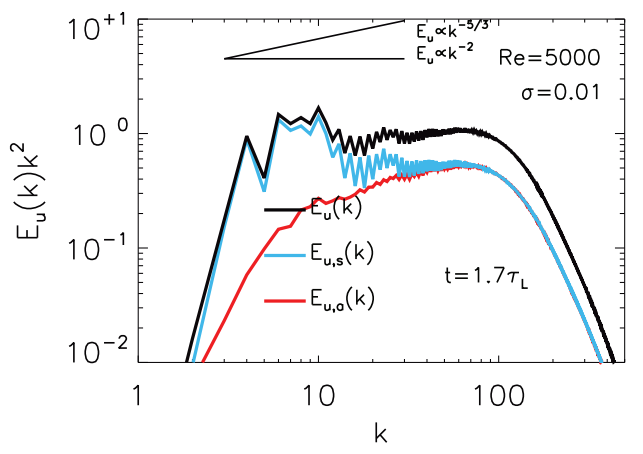

(b)

FIG. 11. (Color online) (a) Magnetic energy and (b) kinetic energy spectra for $\sigma=0.01$ and $\mathrm{Re}=5000$ at the first peak of total dissipation rate $t / \tau_{L}=1.7$.

development of the perturbation has lead to the bending and curling of the current and vorticity sheets. The basic structures, however, remain unaltered without development of additional features. On the other hand, at higher Reynolds number (i.e., $\operatorname{Re}=5000$ and $\sigma=10^{-2}$ ) more structures appear [see Fig. 9(c)].

While the bent current and vorticity sheets are still present, strong fluctuations, reminiscent of structures in flows with random initial conditions, appear at the edge of the sheets due to the development of an instability. Note that these fluctuations have generated scales smaller than the thickness of the "ordered" current and vorticity sheets. These structures are responsible for the second peak of the Ohmic dissipation observed in Fig. 7(a) but also for the peak of the viscous dissipation at later times as Re increases [see Fig. 7(b)]. This observation supports our conjecture that at $\operatorname{Re} \gg 1$ these turbulent fluctuations will dominate and the second peak of $\epsilon_{b}$ will become a global maximum. Finally, in Fig. 9(d) the effect of the perturbation is much more pronounced for the $\sigma=0.1$ and $\mathrm{Re}=2000$ run; not only at the edge of the current and vorticity sheets but also away from the symmetry planes, where strong current and vorticity turbulent structures have emerged. This probably indicates that the amplitude of the perturbation $\sigma=0.1$ was large enough that asymmetric current or vorticity was not only amplified by its interaction with the symmetric part of the flow but also by self-interaction of the structures introduced by the perturbation.

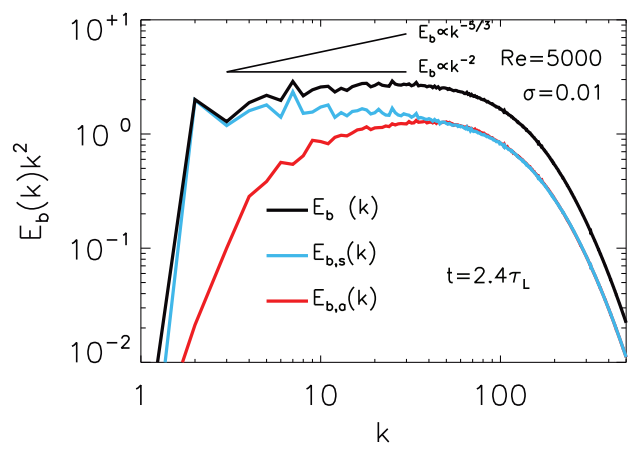

(a)

\section{Spectral behavior}

As we stated in the introduction, it is an open question whether the breaking of the symmetries will change the power law exponent of the spectra. Therefore, in this section we investigate the effect of symmetry breaking on the inertial range scaling of our energy spectra.

In Fig. 10 we show the energy spectra for the $\sigma=0.1$ and $\mathrm{Re}=2000$ case. Figure 10(a) represents the magnetic energy spectrum at the peak of the Ohmic dissipation and Fig. 10(b) the kinetic energy spectrum at the peak of viscous dissipation. The blue (light gray) and the red (dark gray) lines in these figures indicate the symmetric and asymmetric parts of the energy spectra, respectively, whereas the black lines indicate the full spectra, i.e., symmetric plus asymmetric part. At the peak of Ohmic dissipation $E_{b, s}$ and $E_{b, a}$ reach equipartition within the range $20 \lesssim k \leqslant k_{\max }$. The full magnetic energy spectrum compensated by $k^{2}$ has clearly a positive slope and a linear fit indicates a value close to the $k^{-5 / 3}$ scaling. For the kinetic energy spectrum, equipartition occurs between $E_{u, s}$ and $E_{u, a}$ at all scales. The slope of the full compensated spectrum $k^{2} E_{u}$ is positive and also close to $k^{-5 / 3}$. However, the range of wave numbers that exhibit a power law for the velocity field is shorter than for the magnetic field and a distinction between $k^{-5 / 3}$ and $k^{-3 / 2}$ is not possible. Hence, it is clear that for $\sigma=0.1$ the spectrum moved away from the $k^{-2}$ scaling and it returned to the classical $k^{-5 / 3}$ (or $k^{-3 / 2}$ ) turbulence scaling. Note, however, that this case was strongly perturbed at $t=0$

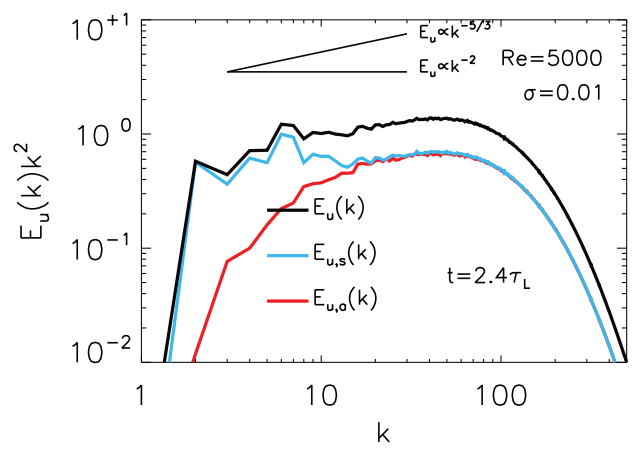

(b)

FIG. 12. (Color online) (a) Magnetic energy and (b) kinetic energy spectra for $\sigma=0.01$ and $\operatorname{Re}=5000$ at the second peak of total dissipation rate $t / \tau_{L}=2.4$. 


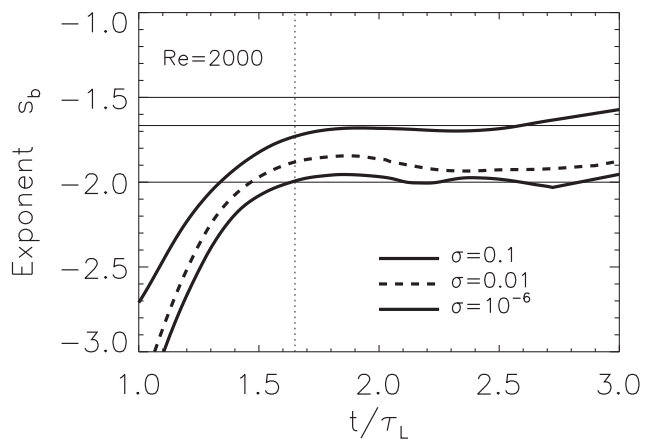

(a)

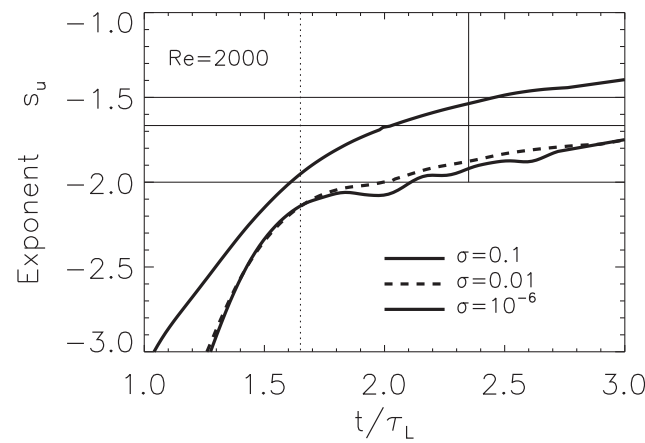

(b)

FIG. 13. Scaling exponent of (a) the magnetic energy and (b) the kinetic energy spectrum for different $\sigma$ and $\operatorname{Re}=2000$.

with a significant amount of enstrophy and current density introduced by the perturbation.

The case with $\sigma=0.01$ and $\operatorname{Re}=5000$ is more insightful since both the energy, enstrophy and square current density of the perturbation are significantly smaller than the TG initial conditions. The spectra for this case are shown for the two total dissipation peaks in Figs. 11 and 12 at times $t / \tau_{L}=$ 1.7 and $t / \tau_{L}=2.4$, respectively. Both magnetic and kinetic energy spectra reach equipartition between the symmetric and the asymmetric part of the flow at small scales but not at large scales. In particular, at the first total dissipation peak $E_{b, s} \sim E_{b, a}$ and $E_{u, s} \sim E_{u, a}$ only for wave numbers $k>30$ (see Fig. 11). The slopes of the compensated full energy spectra $k^{2} E_{b}$ and $k^{2} E_{u}$ are positive but less than the $k^{-5 / 3}$ scaling. Note that for the symmetric part of the magnetic field $k^{2} E_{b, s} \sim$ const. Therefore, $k^{-2}$ is still a good scaling for $E_{b, s}$ implying that the change in the slope of $E_{b}$ is due to the symmetry breaking part of the flow.

In the second dissipation peak more scales have reached equipartition between the symmetric and the asymmetric part of the flow (see Fig. 12). The power law of the full magnetic energy spectrum remains between $k^{-2}$ and $k^{-5 / 3}$ while the full kinetic energy spectrum is closer to $k^{-5 / 3}$.

The evolution of the scaling exponents for the magnetic energy $\left(E_{b} \sim k^{s_{b}}\right)$ and kinetic energy spectra $\left(E_{u} \sim k^{s_{u}}\right)$ for various cases of Table I are presented in Figs. 13 and 14. The scaling exponents were obtained using a linear fit on the energy spectra between wave numbers $4<k<20$ for the runs with $\operatorname{Re}=1000,4<k<30$ for the runs with $\operatorname{Re}=2000$, and $4<k<40$ for the run with $\mathrm{Re}=5000$. We note that measured exponents in this way are sensitive in the choice of the fitting range especially away from $t_{\text {peak }}$. However, the objective here is to show the time evolution of the exponents and not the precise value. Our choices were based on Figs. 10 to 12 as the most reasonable to our opinion. The effect of the perturbation amplitude on the spectral exponents $s_{b}$ and $s_{u}$ is shown in Fig. 13. The strongly perturbed case with $\sigma=0.1$ deviates at early times from the other weakly perturbed cases. It saturates to a value close to $-5 / 3$ for $s_{b}$ while no clear saturation can be observed for $s_{u}$. This indicates that Reynolds number is not high enough for a clear scaling of the kinetic energy. Even in the unperturbed case $E_{u}$ does not have a clear power law scaling (see [16]). The weakly perturbed cases for the magnetic field reach a value close to $s_{b}=-2$ at the peak of total dissipation and saturate close to this value, whereas $s_{u}$ is close to -2 at the total dissipation peak and it drifts to higher values as time progresses.

Figure 14 focuses on the $\sigma=0.01$ case where the highest Re was obtained. Although $s_{u}$ is close to -2 at the peak of the total dissipation $\left(t / \tau_{L} \simeq 1.7\right)$, it increases with time and becomes closer to $-5 / 3$ as $\mathrm{Re}$ increases at the peak of the viscous dissipation [see Fig. 14(b)]. The scaling exponent of the magnetic energy spectrum asymptotes in time to a value between -2 and $-5 / 3$, while an increase with $\mathrm{Re}$ is observed at the second peak of Ohmic dissipation $\left(t / \tau_{L} \simeq 2.4\right)$.

Therefore, our results indicate that in the perturbed system and for high Re the spectral exponent transitions to a value larger than -2 . This can be seen most clearly by comparing

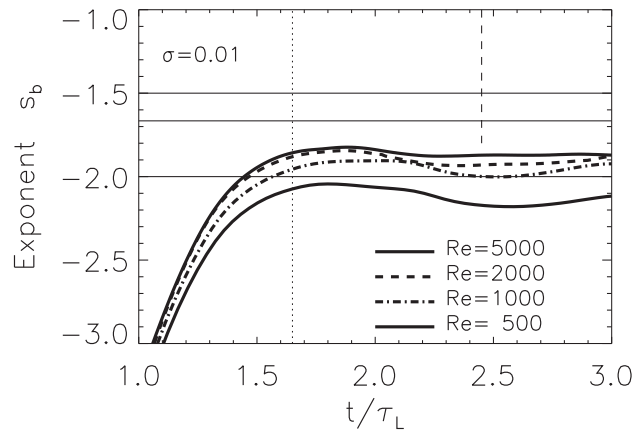

(a)

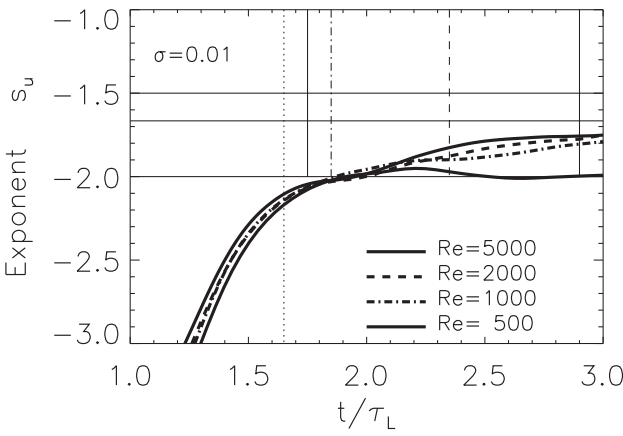

(b)

FIG. 14. Scaling exponent of (a) the magnetic energy and (b) the kinetic energy spectrum for different $\operatorname{Re}$ and $\sigma=0.01$. 


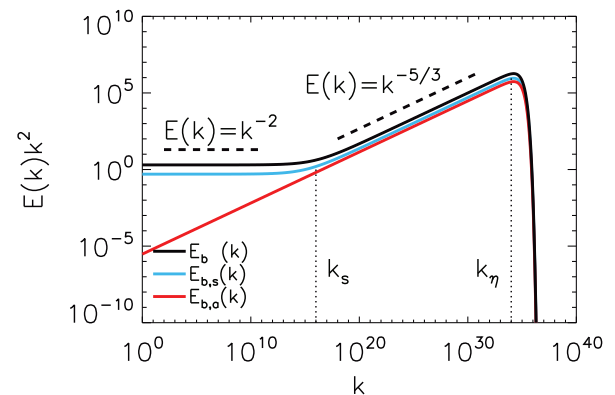

FIG. 15. (Color online) Sketch of an idealized energy spectrum at very high Reynolds number.

the total energy spectrum to the symmetric spectrum, which still follows the $k^{-2}$ scaling in Figs. 11 and 12. A best fit gives a value for the exponent smaller than $-5 / 3$. We conjecture, however, that this intermediate value of the exponent, i.e., $-2<s_{b}<-5 / 3$, is a finite Reynolds number effect. We expect that as Re increases, the small scales that break the symmetries will start forming a strong turbulence scaling (i.e., $k^{-5 / 3}$ or $k^{-3 / 2}$ ). The spectrum in these scales is related to the turbulent fluctuations observed in Figs. 9(c) and 9(d). As Re will increase, more small scale turbulent fluctuations will be excited and consequently the range of validity of this power law scaling will increase. Ultimately, this will be the dominant spectrum as $\operatorname{Re} \rightarrow \infty$. This is what we try to depict in Fig. 15 by presenting schematically the expected energy spectra at Re $\gg 1$. For the large scales where the symmetries are not broken, we expect the $k^{-2}$ energy spectrum, which reflects the presence of the strong current and vortex sheets, to persist. The wave number $k_{s}$, that depends on the amplitude of the perturbation, determines the transition point between the $k^{-2}$ and the $k^{-5 / 3}$ spectrum. If the symmetries are broken at all scales at $\operatorname{Re} \gg 1$, then the -2 power law spectrum could vanish.

At the present resolutions we do not have enough inertial range to distinguish between different power laws, i.e., one for the small and one for the large wave numbers. As a result, the scaling exponent that is observed appears as an intermediate value.

\section{CONCLUSIONS}

In this work we have studied one of the proposed initial conditions in [14] for a freely decaying MHD flow. These initial conditions in the absence of any perturbation lead to the formation of strong magnetic shear layers that result in a $k^{-2}$ energy spectrum [16] different than the more commonly obtained spectra $k^{-5 / 3}$ and $k^{-3 / 2}$ for random initial conditions. Here, we investigated whether this behavior persists when the initial conditions weakly deviate from the ones proposed in [14] and break the involved Taylor-Green symmetries by adding a small perturbation.

We demonstrated that a sufficiently small perturbation evolves passively and it grows at a rate that increases with Reynolds number. In particular, it was shown that the energy ratio scales as $E_{a} / E_{s} \sim \mathrm{Re}$ and the dissipation ratio scales as $\epsilon_{a} / \epsilon_{s} \sim \mathrm{Re}^{3 / 2}$ at the peak of the total dissipation rate. Therefore, for any finite amplitude perturbation, no matter how small it is, there is a high enough Reynolds number for which the perturbation will grow enough at the peak of the total dissipation resulting in a nonlinear feedback in the flow and subsequently break the TG symmetries.

For strong perturbations of amplitude $\sigma=0.1$ we showed that the TG symmetries break. Turbulent small scales appear both near the strong shearing regions but also in the bulk of the $[0, \pi]^{3}$ boxes. These new small scale features change the slope of the energy spectrum from $k^{-2}$ to the classical turbulence spectrum, i.e., $k^{-5 / 3}$ or $k^{-3 / 2}$ power law scaling.

For the smaller amplitude perturbation $\sigma=0.01$ the initially passive asymmetric part of the flow grows to an amplitude that can play a nonlinear role in the MHD equations at large Re. A new dissipation peak appears as a result of the nonlinear evolution of the instability. The strong shearing regions bend and turbulent structures appear at the edge of the current sheet causing this new peak. The scaling exponent of the energy spectrum is clearly larger than -2 but still smaller than $-5 / 3$. This intermediate value of the exponent appears because at the examined Reynolds numbers the small scales have broken the symmetries and are approaching the strong turbulence scaling, while the large scales still exhibit the $k^{-2}$ scaling. Therefore, the measured exponent appears to take an intermediate value. We argue that the strong turbulence scaling (i.e., $k^{-5 / 3}$ or $k^{-3 / 2}$ ) will dominate at higher $\mathrm{Re}$.

The above results suggest that unless the TG symmetries are satisfied exactly in periodic boxes they will break at sufficiently large Re and the strong turbulence scaling of the spectrum will be recovered. Therefore, the debate seems to return back to the distinction between the $k^{-5 / 3}$ and the $k^{-3 / 2}$ that we could not resolve here. Even though the question of universality in MHD turbulence is in general still open, the particular mechanism for the generation of the $k^{-2}$ spectrum of the TG flow seems to be unstable at high Re and is not expected to appear for a general class of flows. It could however appear at intermediate Re.

Consequently, an important issue that this work has brought up is why and under what conditions these large current sheets, which lead to the transient $k^{-2}$ energy spectra, form. Even though current sheets form spontaneously in MHD [31], in this case the spanwise length was the size of the fundamental box and their amplitude was strong enough to dominate the energy spectrum, something that is not typically observed in random MHD turbulent flows. It is crucial in observations to distinguish the $k^{-2}$ spectra that manifest due to discontinuities in the magnetic field and those due to weak turbulence. Note that these mechanisms are distinctly different even though the energy spectra display the same scaling. Some of these questions are going to be addressed in our future work.

\section{ACKNOWLEDGMENTS}

V.D. acknowledges the financial support from EUfunded Marie Curie Actions-Intra-European Fellowships (FP7-PEOPLE-2011-IEF, MHDTURB, Project No. 299973). The computations were performed using the HPC resources from GENCI-TGCC-CURIE (Project No. x2013056421) and PRACE-FZJ-JUQUEEN (Project No. PRA068). 
[1] D. Biskamp, Magnetohydrodynamic Turbulence (Cambridge University Press, 2003).

[2] Y. Zhou, W. H. Matthaeus, and P. Dmitruk, Rev. Mod. Phys. 76, 1015 (2004).

[3] S. Boldyrev, Phys. Rev. Lett. 96, 115002 (2006).

[4] C. S. Ng and A. Bhattacharjee, Phys. Plasmas 4, 605 (1997).

[5] S. Galtier, S. V. Nazarenko, A. C. Newell, and A. Pouquet, J. Plasma Phys. 63, 447 (2000).

[6] W. C. Müller and R. Grappin, Phys. Rev. Lett. 95, 114502 (2005).

[7] P. D. Mininni and A. Pouquet, Phys. Rev. Lett. 99, 254502 (2007).

[8] J. J. Podesta, D. A. Roberts, and M. L. Goldstein, Astrophys. J. 664, 543 (2007).

[9] J. Saur, H. Politano, A.Pouquet, and W. H. Matthaeus, Astron. Astrophys. 386, 699 (2002).

[10] M. Wan, S. Oughton, S. Servidio, and W. H. Matthaeus, J. Fluid Mech. 697, 296 (2012).

[11] A. A. Schekochihin, S. C. Cowley, and T. A. Yousef, in IUTAM Symp. Comput. Phys. and New Perspectives in Turb., IUTAM Bookseries, Vol. 4, edited by Y. Kaneda, G. M. L. Gladwell, and R. Moreau (Springer, 2008), pp. 347-354.

[12] P. D. Mininni, Annu. Rev. Fluid Mech. 43, 377 (2011).

[13] R. Grappin, J. Leorat, and A. Pouquet, Astron. Astrophys. 126, 51 (1983).

[14] E. Lee, M. E. Brachet, A. Pouquet, P. D. Mininni, and D. Rosenberg, Phys. Rev. E 81, 016318 (2010).

[15] A. Beresnyak and A. Lazarian, Astrophys. J. Lett. 722, L110 (2010).

[16] V. Dallas and A. Alexakis, Phys. Rev. E 88, 053014 (2013).

[17] M. E. Brachet, D. I. Meiron, S. A. Orszag, B. G. Nickel, R. H. Morf, and U. Frisch, J. Fluid Mech. 130, 411 (1983).
[18] E. Lee, M. E. Brachet, A. Pouquet, P. D. Mininni, and D. Rosenberg, Phys. Rev. E 78, 066401 (2008).

[19] V. Dallas and A. Alexakis, Phys. Fluids 25, 105106 (2013).

[20] J. E. Stawarz, A. Pouquet, and M.-E. Brachet, Phys. Rev. E 86, 036307 (2012).

[21] D. Gottlieb and S. Orszag, Numerical Analysis of Spectral Methods: Theory and Applications, Vol. 26 (SIAM, 1977).

[22] M. Frigo and S. Johnson, in Acoustics, Speech, and Signal Processing, 1998, Proceedings of the 1998 IEEE International Conference (IEEE, 1998), Vol. 3, pp. 1381-1384.

[23] D. O. Gómez, P. D. Mininni, and P. Dmitruk, Adv. Space Res. 35, 899 (2005).

[24] P. D. Mininni, D. Rosenberg, R. Reddy, and A. Pouquet, Parallel Computing 37, 316 (2011).

[25] G. Taylor and A. Green, Proc. R. Soc. London A 158, 499 (1937).

[26] M. D. Bustamante and M. Brachet, Phys. Rev. E 86, 066302 (2012).

[27] Y. Ponty, P. D. Mininni, J.-P. Laval, A. Alexakis, J. Baerenzung, F. Daviaud, B. Dubrulle, J.-F. Pinton, H. Politano, and A. Pouquet, C. R. Phys. 9, 749 (2008).

[28] S. Oughton, M. Wan, S. Servidio, and W. H. Matthaeus, Astrophys. J. 768, 10 (2013).

[29] A. Pouquet, E. Lee, M. Brachet, P. Mininni, and D. Rosenberg, Geophys. Astrophys. Fluid Dyn. 104, 115 (2010).

[30] G. Krstulovic, G. Thorner, J.-P. Vest, S. Fauve, and M. Brachet, Phys. Rev. E 84, 066318 (2011).

[31] E. N. Parker, Spontaneous Current Sheets in Magnetic Fields: With Applications to Stellar X-Rays (Oxford University Press, 1994).

[32] E. Priest and T. Forbes, Magnetic Reconnection: MHD Theory and Applications (Cambridge University Press, 2007). 